\title{
Developing a methodological approach for interpreting and predicting pedestrian movement in a public space for sustainable urban planning
}

\author{
K. Monokrousou \& M. Giannopoulou \\ Department of Civil Engineering, \\ Democritus University of Thrace, Greece
}

\begin{abstract}
Improving quality and standards of living of the urban environment has always been a challenge for urban planners. The modern perception of urban planning is directed towards promoting pedestrian movement in the urban space. Thus, understanding how this is generated and functions in relation to the urban layouts as well as predicting the effects of pedestrian movement with regards to the location of the various land uses are key elements for sustainable urban planning. This paper intends to interpret the characteristics of open urban space to create, through more holistic approaches, a methodological framework for decisionmaking analysis towards sustainable urban planning. In a first approximation, this framework consists of space syntax theory that can effectively study the configuration characteristics of urban space. A further step is to incorporate the role of land uses in the pedestrian movement patterns. Land uses tend to increase pedestrian flows in the local area beyond what their syntactic location and properties within the spatial system of the larger area may suggest. The purpose is to test a dynamic tool that can be used to formulate a sustainable urban strategy as well as propose solutions and interventions that improve the structure and quality of the urban system. The case of study is Athens Municipality.

Keywords: syntactic analysis, pedestrian movement, land use effect, sustainable urban planning.
\end{abstract}




\section{Introduction and background}

For the analysis and interpretation of the urban environment, a range of tools and methods can be applied for mapping the profile of the urban area and proposing a framework for urban planning in accordance with the principles of sustainable development.

In the modern perception of urban planning, the promotion and encouragement of pedestrian movement in the urban space plays a vital role. Thus, for understanding how pedestrian movement is generated in relation to the urban layouts and for predicting this movement in public spaces, various tools have been applied in a range of studies.

According to the literature, on a large sample of cities different in form, structure, size and culture, the space syntax theory has efficiently interpreted and predicted pedestrian flows as spatial configuration correlates powerfully with observed movement by pedestrians. In several cases, even a proportional relationship is established between the most important space syntax parameter, i.e. integration and pedestrian movement density (Talav Era [1], Law et al. [2], Topçu et al. [3], Read [4], Hillier et al. [5], Peponis et al. [6], Hillier et al. [7]). Other studies show that also other factors influence the choices of pedestrian movement e.g. land use, diversity of land use, connectivity, population densities, average building height (Choi and Koch [8], Choi and Sardari Sayyar [9], Fatah Gen Schieck et al. [10], Özbil and Peponis [11]).

This paper attempted to perform syntactic analysis to investigate this correlation as well as incorporate the land use effect. The overall purpose is to interpret effectively the densities of pedestrian movement in order to develop a methodological decision-making approach for sustainable urban planning.

The case of study is the Municipality of Athens, in Greece, as this area is a very dense system in terms of population and quite complex in terms of land use mix.

\section{Methodology}

\subsection{The case of study: Athens Municipality}

Athens is the capital of Greece and the Municipality of Athens is the largest municipality of the country with an area of about 39 square kilometres and inhabitants of about 665 thousand. The population density of the municipality is about 17,000 inhabitants per square kilometer, which makes is by far one of the densest urban areas in the developed world.

The tissue of Athens Municipality has been developed from antiquity until now around the various city centers and the city's several hills in a very dense and complex manner (Figure 1). The postwar urban, socio-economic and population development of Athens resulted to the current unbalanced over accumulation of population, activities and services. Consequently, in the greater metropolitan area of Athens almost half of the population of Greece has been accumulated resulting in a significant spatial change on the city's landscape. The explosive inhabitants 
rise along with the over exploitation of public space led to a morphology of very tight urban tissue and an increased use of automobiles.

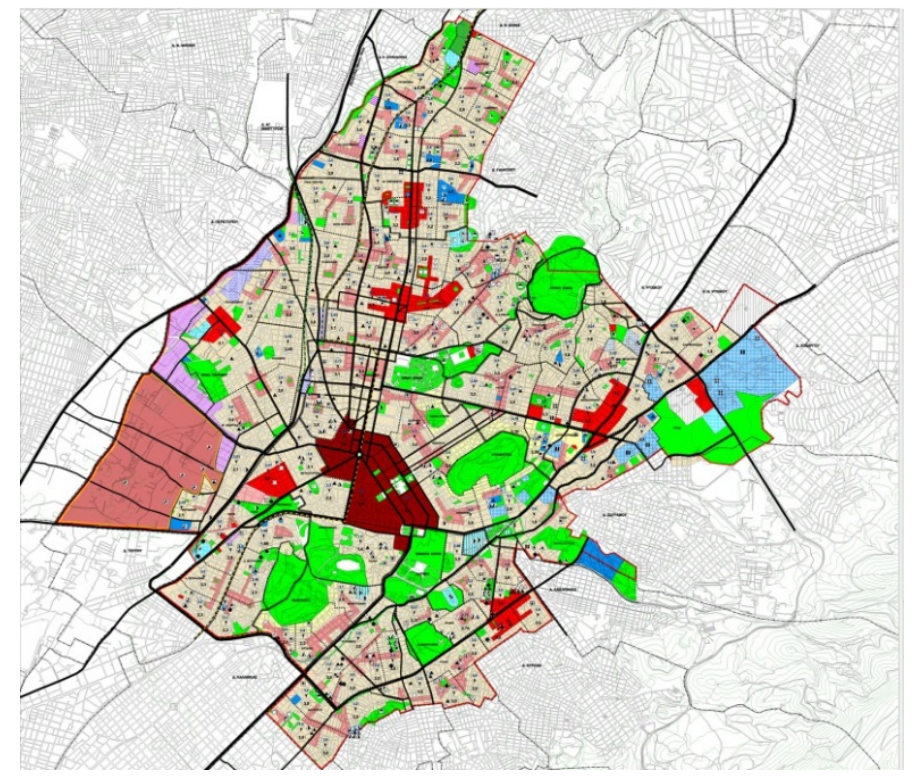

Figure 1: $\quad$ Master Plan for Athens, Attica, (proposal), 2011 (ORSA [12]).

The street network that has been developed over the years has both the typical characteristics of the traditional tissues in parts of the city center and a linear development of the major roads that connect the city center with the periphery. This configuration resulted in the construction of additional roads and parking spaces instead of investing in improving public transportation services. Besides, the lack of fixed track transport encouraged even more the use of private cars, leading to an excessive increase of traffic volume. In this respect, Athens Master Plan aims at changing the current trends and strengthening the role of public transport and pedestrian movement.

\subsection{Steps of the procedure and data}

To interpret and predict the densities of pedestrian movement, syntactic analysis was performed as a first approach in the study area.

The axial map was automatically generated, using an open source extension of ArcGIS 10.2, the Axwoman 6.3 (Axwoman [13]). The axial map was then shaped and every street was represented by an axis in the maps (figure 2). Subsequently, the axis lines are imported to the open-source software platform DepthmapX (DepthmapX [14]) to perform a complete analysis of the different space syntax 
parameters. Connectivity, integration and choice are among the variables that were calculated through the DepthmapX software.

The study area was then simulated as a system of "attractors" i.e. land uses that attract pedestrian movement, in order to consider other spatial factors influencing the choices of pedestrians and to eliminate the inefficiency of space syntax in the prediction of pedestrian movement densities.

Land uses were classified into different categories and quantification of the measure of attraction was performed through particular "weight" values according to the significance of each attractor. The measure of attraction ranged between 1 and 100, with the value of 1 corresponding to about 1,000 visitors per week, and the value of 100 corresponding to 100,000 week visitors. Similarly, regarding the level of influence, the attractors were classified according to their effect between two categories: (a) long range attractors with effect beyond 500 meters and (b) local attractors.

Mixed syntax software was used (Syrigos [15]) to calculate the proximity cores of the spatial system for every attractor category. The total proximity core was calculated for all the attractors' categories, integrating the different cores into one map. Conclusions were made both for the individual proximity cores of each attractor category and for the total proximity core.

The production of the synthetic integration map was produced by incorporating the integration map of space syntax $(75 \%)$ to the proximity core $(25 \%)$ after normalising these values. With this final step the inconsistencies on the density of pedestrian movement were eliminated and a more solid prediction was performed.

For the urban street network, open street data were obtained by Open Street Map (OSM [16]). Land uses data were similarly obtained by the Open Street Map database and were complemented by the ArcMAP OpenSteetMap basemap along with land use data of Athens Municipality maps. Data regarding the average visitors per week were collected for different land use categories in a representative manner. For the rest of the land uses, extrapolations were made based on empirical observation. Additionally, the table of classification and calibration of attractors by Gospodini and Syrigos [17] was used as reference values.

The selection of percentages for the synthetic integration map originated from experiential observations that better reflect Athens Municipality.

\section{Results}

Following the methodology, the axial map of Athens Municipality was automatically generated (figure 2).

The global integration map of the municipality was produced (figure 3 ) through axial analysis in DepthmapX, the red lines illustrating the streets with the highest integration values, while the blue ones the most segregated ones. However, the correlation between integration values and pedestrian movement densities is not strong for particular spaces of the study area according to empirical observation of the street network. 


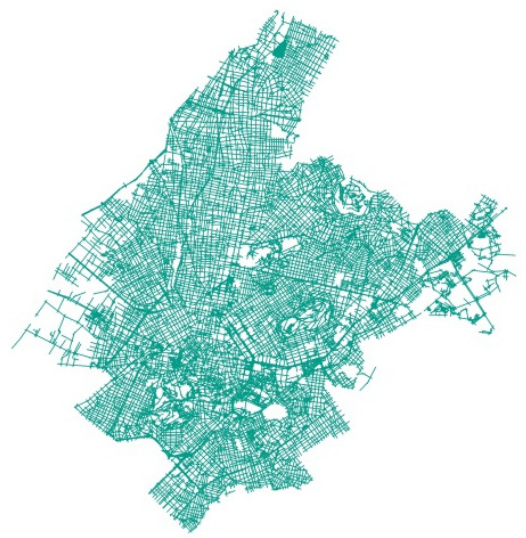

Figure 2: Axial map of Athens Municipality.

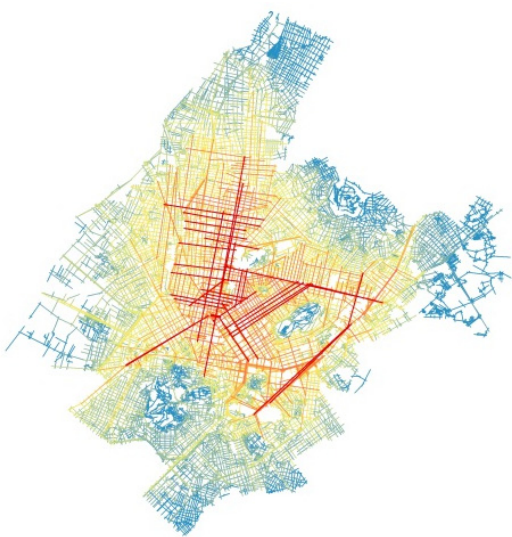

Figure 3: Global integration map of Athens Municipality.

To implement the approach of "land uses as attractors", land uses were added in ArcMAP as points corresponding to building level. For the case of study, land uses exceeded the quantity of 3.300 and a classification into nine (9) categories was conducted. Every attractor received a value for the level of attraction (1-100) and a value for the type of influence, global (0) or local (1).

In Mixed Syntax, data of the system axial map as well as the attractors of each category was added in order to produce the proximity cores for each attractor category separately (figure 4).

Regarding the location, size and form of the proximity cores, some initial conclusion can be drawn:

- In respect to leisure activities (figure 4(a)), the spatial system generates a short proximity core with circular developement around the historical and business center. Although Athens has an extented development of leisure activies, it seems that the majority and more important places are gathered within a rather narrow and robust core.

- Concerning important public buildings and services (figure 4(b)), a rather extensive proximity core is generated expanding slightly towards the northeast part of the study area, where the largest administration units (i.e. courts) of the city are hosted. It is indicative that the average number of week visitors in the courts is about 125.000 .

- Regarding commerce (figure 4(c)), the spatial system generates one strong and concrete proximity core. In this respect, the major commerce units are seem to be accumulated into a narrow area of the city centre. In this respect, the periphery of the municipality appears to be having a disadvantage in comparison to the city center.

- As far as cultural institutions and churches are concerned (figure 4(d)), a more extensive proximity core is generated expanding slightly towards the southwest part of the study area, where the major cultural institutions and historical 
monuments are located (e.g. the Acropolis museum). Other attractors of this category include theatres, opera, cinemas as well as churches that are spread quite evenly in the area of study. Thus, the municipality appears as a symmetric spatial system in terms of culture.

- In regards to tourist services (figure 4(e)), one proximity core is generated expanding slightly towards the southwest part of the area, towards Syntagma and Acropolis, where the majority of the largest hotels are based. Given the fact that the historical centre has geographically central location, the city appears again a symmetric spatial system in terms of tourist services.

- With regards to transportation services (figure 4(f)), there are various proximity cores scattered in the area of study as well as a linear development along the metro lines. Two strong cores are located in the area of the Larisis central train station as well as the Liosion central bus station. Other proximity cores appear in the more central and popular metro stations of the city (Omonia and Syntagma). Less important transportation attractors are located in other metro stations as well as the bus station in Pedion Areos for transportation in the region of Attica.

- Regarding universities and schools (figure 4(g)), three strong proximity cores are generated. One in the heart of the city, where part of the polytechnic school, the university of economics and business as well as the law school of Athens are located. The other core is generated in the east part of the area, where, among other institutions, the medical school of Athens is based. The last significant core is found in the west part of the study area where the agricultural university of Athens is located. Other attractors of this category include schools and institutions that are spread quite evenly in the municipality. Thus, the city appears as a symmetric spatial system in terms of schools and only the universities create the unbalanced cores.

- Concerning hospitals and clinics (figure 4(h)) the proximity cores are unsymmetrically spread over the east part of the municipality, where the majority of the largest hospitals are located. The west part of the municipality, which includes the low income neighbourhoods, seems to be less beneficial in terms of health facilities.

- As far as the athletic installations are concerned (figure 4(i)), there are several small and dispersed proximity cores, which cover the central area of study and some south and east areas, that appear to be beneficial in terms of athletic facilities.

The total proximity core is produced i.e. the proximity core counting for all the nine (9) categories of attractors simultaneously (figure 5(b)). In this map the historical centre as well as the CBD appear to be the most beneficial areas. 


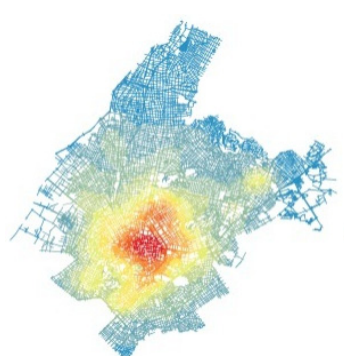

(a)

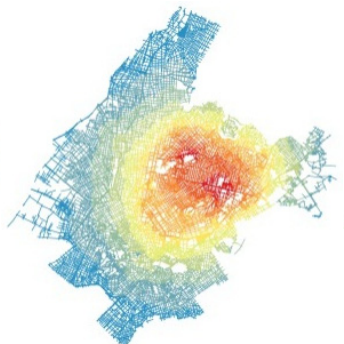

(b)

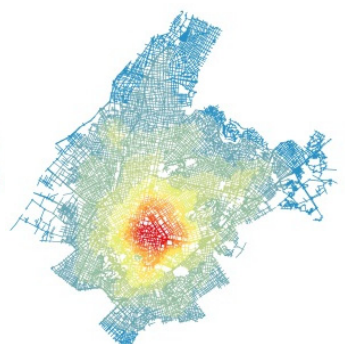

(c)

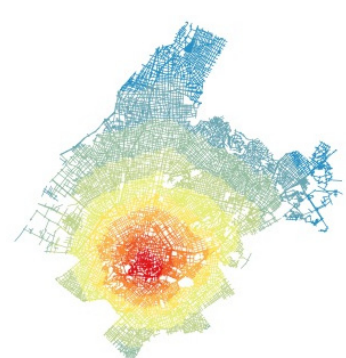

(d)

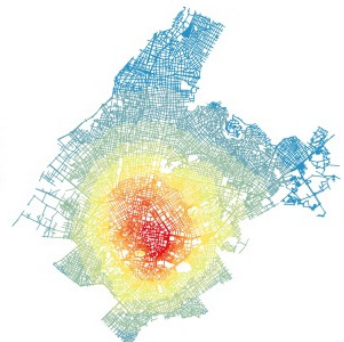

(e)

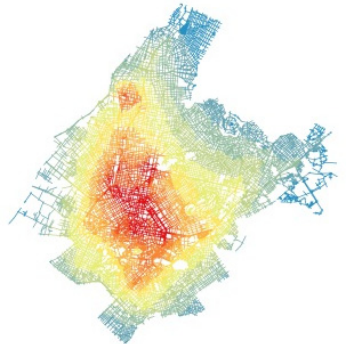

(f)

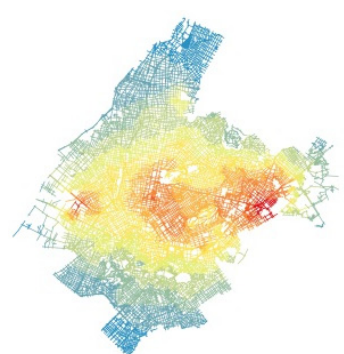

(g)

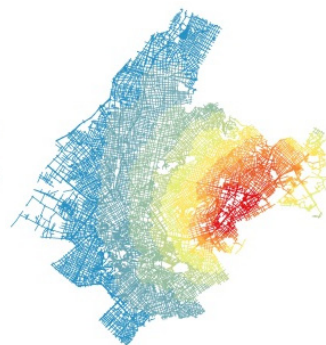

(h)

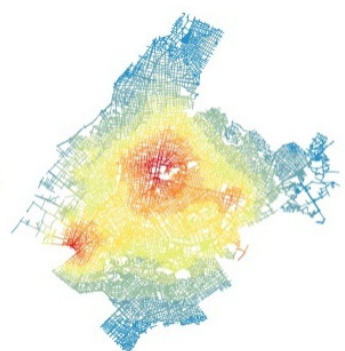

(i)

Figure 4: Proximity cores of Athens Municipality for each attractor category: (a) Leisure activities; (b) Important public buildings and services; (c) Commerce; (d) Cultural institutions - churches; (e) Tourist services; (f) Transportation services; (g) Universities and schools; (h) Hospitals and clinics; (i) Athletic installations. 


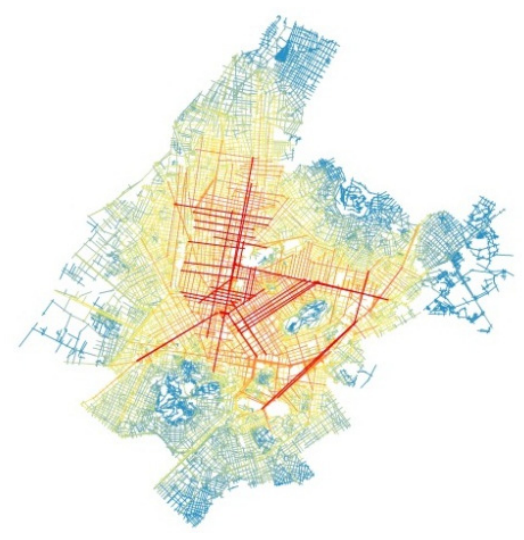

(a)

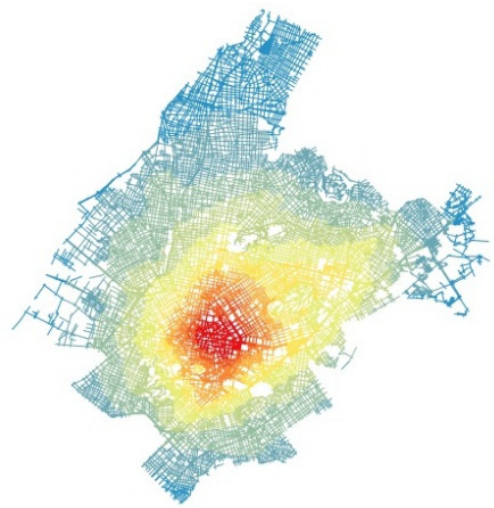

(b)

Figure 5: (a) Integration core; (b) Proximity core (all attractors' categories).

The proximity core, in contrast with the integration core, is less extensive and more localized in the city center, due to the increased number of attractors in the central areas. In this respect, the city seems to be a strongly centralized spatial system offering mainly to the centre a dense network of facilities and services of all kinds.

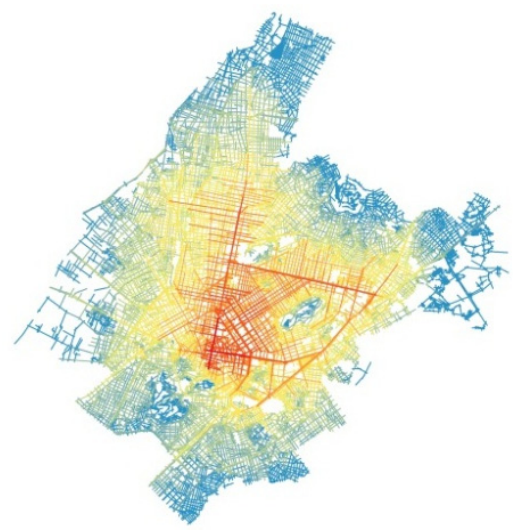

Figure 6: The synthetic integration core.

The synthetic integration core is produced (figure 6) from the normalized values of integration core $(75 \%)$ and proximity core $(25 \%)$, resulting in a more realistic depiction of the density of pedestrian movement. 


\section{Discussion and further research}

The analysis showed that integration values originating from syntactic analysis not always reflect the densities of the pedestrian movement. For instance, the comparatively low integration values that are observed in significant commercial pedestrian streets (e.g. Ermou) or important street in terms of tourism attraction (e.g. Makriyanni, Dionysiou Aeropayitou), cannot fully interpret the pedestrian flows. Additionally, there are streets in the area's periphery where the integration values are low (figure 3), although they exhibit quite high densities of pedestrian movement (e.g. the areas of Metaxourgio, Panormou, Goudi). These streets host specific land uses and activities that attract pedestrians, e.g. leisure activities, administration services.

An improvement in the prediction of the pedestrian movement densities was recorded when the factor of land uses that act as attractors was taken into consideration. For instance, the comparatively low integration values that were estimated for various popular streets with a significant amount of attractors, increased significantly when the attractors approach was implemented. In particular, Karagiorgi Servias, which is a central street in the Syntagma square, appears to be 49th in the hierarchy of the integration core; while in the synthetic integration core, this street climbs up in the 14th position, a rank matching much better with the empirical densities of pedestrian movement.

As a result, in this study it appears that the synthesis integration core seems to better reflect the city based on experiential observations. However, due to the fact that field work on observation and surveying the densities was quite limited, further research is required. Field work in terms of observation of pedestrian movement in a targeted sample of streets though a strategic choice of "gates" of the study area is necessary in order to verify the simulation results. Finally, of course, testing the simulations in other areas of study is important to establish the validity of the methodological tool.

\section{Acknowledgement}

Acknowledgement is made to the State Scholarship Foundation - IKY that support financially the $\mathrm{PhD}$ thesis through the 'IKY Fellowships of Excellence for Postgraduate Studies in Greece - SIEMENS PROGRAM'.

\section{References}

[1] Talav Era R. Improving pedestrian accessibility to public space through Space Syntax Analysis, Proceedings of the 8th International Space Syntax Symposium Santiago, PUC, Paper ref no 8223. pp. 1-16, 2012

[2] Law S., Chiaradia A., Schwander C. Towards a multi-modal Space Syntax analysis, Proceedings of the 8th International Space Syntax Symposium Santiago, PUC, Paper ref no 8021. pp. 1-20, 2012. 
[3] Topçu, M., Topçu, K.D., Kubat A.S., Movement Economy Dependent of Urban Design, Proceedings of the 6th International Space Syntax Symposium, İstanbul. pp. 1-6, 2007.

[4] Read S. Flat City: space syntax derived urban movement network model, Proceedings of the 5th Space Syntax Symposium, Delft, 2005.

[5] Hillier, B., Penn, A., Hanson, J., Grajewski, T., Xu, J. Natural movement or configuration and attraction in urban space use, Environment and Planning B: Planning and Design, Pion, Brondesbury, Vol. 20, pp. 29-66, 1993.

[6] Peponis J., Hadjinikolaou E., Livieratos C., Fatouros D.A. The Spatial core of urban culture. Ekistics, n. 334-335, pp. 43-55, 1989.

[7] Hillier B., Burdett R., Peponis J., Penn A. Creating Life: or does architecture determine anything? Architecture and Behaviour, special issue on the work of the Unit for Architectural Studies, Bartlett School of Architecture and Planning, University College London, pp. 233-250, 1987.

[8] Choi, E., Koch, D. Movement and the connectivity of streets: A closer look at route distribution and pedestrian density, Proceedings of the 10th International Space Syntax Symposium, pp. 1-11, 2015

[9] Choi, E. Sardari Sayyar, S. Urban diversity and pedestrian behavior Refining the concept of land-use mix for walkability. Proceedings of the 8th International Space Syntax Symposium, Paper ref no. 8073, pp. 1-15, 2012

[10] Fatah Gen Schieck, A., Briones, C., Mottram C. Exploring the Role of Place within the Urban Space: The Urban Screen as a socialising platform. Mediacity: Situations, Practices and Encounters, by Frank Eckardt et al. ISBN 978-86596-182-2. pp. 285-294, 2008.

[11] Özbil, A., Peponis, J. Modeling Street Connectivity and Pedestrian Movement According to Standard GIS Street Network Representations. Proceedings of the 6th International Space Syntax Symposium, İstanbul. pp. 1-10, 2007.

[12] Organization for the Master Plan and Environmental Protection of Athens (ORSA), Strategic Plan for Athens - Attica 2021, 2011.

[13] Axwoman 6.3 (http://fromto.hig.se/ bjg/Axwoman.htm)

[14] DepthmapX (www.spacesyntax.net/software)

[15] Syrigos S. Mixed Syntax software product, free for academic and research purposes, developed for the purposes of the research programme "Spatial analysis and architectural interventions in the broader area of Thessaloniki Municipality", scientific responsible: Prof. Kotsiopoulos A., AUTh Professor in the Department of Architecture, 2008.

[16] Open Street Map - OSM (www.openstreetmap.org)

[17] Gospodini A., Syrigos S. Combining Space Syntax and Land-uses for the description of urban space. Proceeding of the Conference on "Planning \& Complexity", AESOP thematic group, Politecnico di Milano - Dipartimento di Architettura, Milan, 22-23/2/2008. 